\title{
University Cooperation Platform (UCP) between Christian-Albrechts-University Kiel (Germany) and Chiang Mai University (Thailand): implementation of image-guided gynecological brachytherapy
}

\author{
Razvan Galalae, MD, PhD 1,2, Ekkasit Tharavichitkul, MD³, Somsak Wanwilairat, PhD³, Imjai Chitapanarux, MD³. \\ Bernhard Kimmig, MD, PhD ${ }^{4}$, Jürgen Dunst, MD, PhD ${ }^{4}$, Vicharn Lorvidhaya, MD, PhD 3.5 \\ 'Medical Faculty, Christian-Albrechts-University Kiel, Germany, ${ }^{2}$ Radiotherapy Department, Evangelical Clinics Gelsenkirchen, Germany. \\ ${ }^{3}$ Radiotherapy Department, Chiang Mai University, Thailand, “Radiotherapy Department, Christian-Albrechts-University Kiel, Germany. \\ ${ }^{5}$ Faculty of Medicine, Chiang Mai University, Thailand
}

\begin{abstract}
Starting in 1999, the University Cooperation Platform (UCP) implemented an exchange program of researchers and clinicians/physicists between the Christian-Albrechts-University Kiel in Germany and Chiang Mai University in Thailand, to initiate a sustainable base for long-term development of image-guided brachytherapy and in general for high-technology radiotherapy in Chiang Mai. A series of UCP protocols, based constructively on each other, were performed and evaluated at intermediate term follow-up. The first protocol, addressing computed tomography (CT)-optimized brachytherapy for advanced cervical cancer $(n=17)$, showed a significant reduction of $\mathrm{D}_{2 \mathrm{cc}}$ for the bladder and sigmoid $(p<0.001)$ while maintaining a very high dose in $\mathrm{D}_{90}$ high-risk clinical target volume (HR-CTV) in comparison with standard point-based planning. In addition, after a follow-up of 19 months no tumor relapse was observed. The second UCP protocol, testing the impact of magnetic resonance imaging (MRI) guidance $(n=15)$ in patients with cervical cancer, proved significantly smaller $\mathrm{D}_{2 c c}$ doses for the bladder, rectum, and sigmoid $(p=0.003$, $p=0.015$, and $p=0.012$ ), and secured highly curative mean doses in $\mathrm{D}_{90}$ HR-CTV of 99.2 Gy. The acute and late toxicity was excellent without any observed grade 3 or higher morbidity. In the third protocol, the combination of image-guided brachytherapy (IGBT) and whole pelvis intensity-modulated external beam radiotherapy (WP-IMRT) $(n=15)$ reaffirmed the significant reduction of $\mathrm{D}_{2 \mathrm{cc}}$ doses for the bladder, rectum, and sigmoid $(p=0.001$ or $p<0.001)$ along with high equivalent dose at 2 Gy (EQD2) in the HR-CTV, and demonstrated very low acute therapy-related toxicity in absence of grade 3 morbidity. The implementation of transabdominal ultrasound (TAUS) was the focus of the fourth UCP project aiming a more generous potential use of image-guidance on long-term, and enhancing the quality of soft tissue assessment complementary to conventionally planned gynecological brachytherapy. Analyses in 29 patients revealed significantly reduced OARs doses in bladder with a total EQD2 > 80 Gy for bladder in only $17.2 \%$ versus $62.1 \%$ in conventional planning, and in rectum EQD2 > 75 Gy in $44.8 \%$ versus $79.3 \%$, respectively.

In conclusion, analyses revealed excellent results for the high-dose-rate IGBT in patients with advanced gynecological cancer both by using CT and MRI, and/or the combination with WP-IMRT. They also define MRI as gold standard for soft tissue assessment and to determine more accurately HR-CTV. The use of TAUS-guidance adds quality aspects to the "classical" conventional X-ray based planning, especially in terms of real-time measures and adequate soft tissue information, and may lower significantly the dose in OARs. The review of all UCP-results reconfirms the importance of the established program that will continue to operate with subsequent projects.
\end{abstract}

Key words: brachytherapy, cervical cancer, computed tomography, gynecological neoplasms, image-guided brachytherapy.

\footnotetext{
Address for correspondence: Razvan Galalae, MD, PhD, Medical Faculty, Christian-Albrechts-University Kiel, Germany, Evangelical Clinics Gelsenkirchen, Munckelstr. 27-32, 45879 Gelsenkirchen, Germany, phone: +49 209-160-53001, « e-mail: wegner@evk-ge.de

Received: 03.02.2015 Accepted: 19.02.2015

Published: 28.02.2015
} 


\section{Purpose}

Despite the introduction of vaccination against human papilloma virus (HPV), cervical cancer is still the most common gynecologic cancer in the world [1] and the most common malignancy in Thai women with an annual incidence in Chiang Mai of 29.7 per 100000 [2]. The outcomes of a series of randomised controlled trials (RCT) define the standard treatment of locally advanced cervical carcinoma as concurrent cisplatin-based chemo-radiotherapy [3-7]. Radiobiological research findings confirm that combining chemotherapy and radiotherapy improves the therapeutic ratio by inhibiting the repair of sublethal damage from radiation, synchronizing cells to a particularly radiosensitive phase of the cell cycle, and by additive direct cytotoxic effects [8].

However, intracavitary brachytherapy (ICBT) is still considered an indispensible part of the composite radiotherapy treatment for patients with locally advanced cervical cancer, contributing to excellent local control rates of $85 \%$ or higher [9] by the application of very high total prescribed doses between 80 and 85 Gy EQD2 (biologically equivalent dose in $2 \mathrm{~Gy}$ fractions). The diagnosis of cervical cancer is associated with irregular participation in national screening programs for secondary prevention [10], while the primary prevention by systematic vaccination against human papilloma virus remains largely a privilege of the industrialized countries. However, the resources in terms of screening, HPV vaccination, diagnosis, staging, and treatment are limited in developing countries. Thus, it is necessary to design and implement local customized high-technology programs in order to improve the cancer care and outcomes in the regional/national population adapted to the local conditions of a developing country.

\section{Design and introduction of a tailored cooperation platform}

Starting in 1999, a University Cooperation Platform (UCP) was founded between the Christian-Albrechts-University Kiel (Germany) and Chiang Mai University (Thailand). This program was initiated as a two-institution initiative with following primary goals: 1) to develop a University Center of Excellence focused on high-technology brachytherapy for cervical cancer in Chiang Mai (Thailand); 2) to implement an exchange program of researchers and clinicians/physicists between the two institutions; 3) to create a sustainable base for long-term development of image-guided brachytherapy in Chiang Mai; 4) to ensure a long-term Two-Institution-Cooperation Program, which could then be expanded to other fields of high-technology radiotherapy.

In addition, further specific problems had to be considered and addressed: 1) very high cervical cancer patient volume per year (> 250 patients) in Chiang Mai; 2) limited resources in terms of high work load per physician and high patient/physician ratio; 3) limited resources in terms of patient-side financial restrictions including on-site availability favoring short-term medical interventions (e.g. brachytherapy); 4) initial limited technical resources (hard- and software) in Chiang Mai.
In general, this UCP initiative should ensure a transfer of know-how and scientific, technical, and clinical expertise from Christian-Albrechts-University Kiel (Germany) to Chiang Mai University (Thailand), but also ease the transmission of very high level clinical expertise from Chiang Mai in the opposite direction in the best sense of a globalized state-of-the-art personalized cancer care. At advanced status of cooperation, the UCP initiative was aimed to facilitate the creation of an eye-to-eye research platform, and to conduct clinical trials based in both institutions in order to achieve and validate new scientific knowledge. Obviously, the new data should be jointly analyzed and published, and the added knowledge implemented into clinical routine in a rational way with the highest clinical benefit for the majority of the patients to be treated in the context of the two involved institutes of maximum level of cancer care.

\section{Preparatory activities and technical developments}

Starting 1999, the exchange program of researchers and clinicians/physicists between the two institutions has been implemented. Physicians/radiation oncologists (e.g. Kamnerdsupaphon P, Tharavichitkul E, Panichevaluk A) and physicists (e.g. Wanwilairat S), and several PhD students from Chiang Mai University (Thailand) have performed on-site clinical/research fellowships at the Christian-Albrechts-University Kiel (Germany) (range between 2 and 18 months). A series of on-site visits (range 2-4 weeks) have been also performed from University of Kiel researcher/radiation oncologists (e.g. Hebbinghaus D, Jensen M, Galalae R) at Chiang Mai University in Thailand. In addition, numerous congresses and meetings were jointly performed and/or attended (e.g. Thai Society of Therapeutic Radiology and Oncology THASTRO annual meetings Chiang Rai 1999, and Ayutthaya 2003/Symposium "3D Radiotherapy" in Chiang Mai 2003/KIEL TALKS 2006 and 2008 etc.). In addition, a modern CT scanner and two modern linacs along with state-of-the-art treatment planning systems for external beam and brachytherapy were installed in a new section building of the radiotherapy department at Chiang Mai University as a technical prerequisite for further developments [11].

\section{Pre-University Cooperation Platform studies}

From August 1999 to December 2004, a single centre, randomised, non-inferiority trial was conducted at Radiotherapy Department of Chiang Mai University comparing concurrent chemoradiotherapy with carboplatin versus standard concurrent chemoradiotherapy with cisplatin in patients with locoregionally advanced nasopharyngeal cancer (NPC) [12]. Two hundred-six patients with locally advanced NPC were randomised with 101 to cisplatin arm and 105 to carboplatin arm. With a median follow-up of 26.3 months (range: 3-74.6 months), the compliance was superior in the carboplatin arm with $59 \%$ of patients in the cisplatin patient group completing the planned concurrent chemoradiation treatment, compared to $73 \%$ in the carboplatin arm. In addition, the treatment related 
morbidity was also superior in the carboplatin arm with more renal toxicity, leucopenia, and anaemia in the cisplatin group, but more thrombocytopenia in the carboplatin arm. The outcomes in terms of 3-year disease free survival rates were not different with $63.4 \%$ for the cisplatin group and $60.9 \%$ for the carboplatin group $(p=0.9613)$. The same was true for the 3-year overall survival rates with $77.7 \%$ and $79.2 \%$ for cisplatin and carboplatin groups, respectively $(p=0.9884)$. Thus, the treatment efficacy did not differ in both study arms, the carboplatin schedule being associated with lower toxicity. This information is important in the context of high co-morbidity within the local population in North Thailand and could be transferred to cervical cancer as well.

\section{University Cooperation Platform preparatory studies}

A common clinical dilemma is the management of therapy compliance failure when involved in therapy decisions according cohorts of patients stressed with co-morbidities. Therefore, a retrospective study was performed to analyze the impact of an incomplete weekly cisplatin plans within the concurrent standard chemoradiotherapy in 165 consecutive treated patients with locally advanced cervical cancer. Ninety-two patients (55\%) completed the initially prescribed protocol of 6 cycles of weekly cisplatin. After a follow-up of 3 years, the local control rate was significantly superior with $95.4 \%$ in the patients with 6 completed cycles versus $<6$ cycles with $84.8 \%(p=0.028)$. However, disease-free $(p=0.22)$ and metastases-free (0.88) survival rates did not differ significantly [13]. A further trial was conducted to compare different used historical brachytherapy fractionations [14].

\section{First University Cooperation Platform major project - introduction of computed tomography-based image guided cervix brachytherapy}

Intracavitary brachytherapy has been used in the form of high-dose-rate (HDR) brachytherapy (BT) for the treatment of cervical cancer over 40 years in oder to escalate the dose to very high total radiation doses in the tumor region, while optimally sparing the adjacent normal organ tissues, and thus, lowering the integral dose [15]. High-dose-rate BT is the preferred brachytherapy method in Europe and Asia where it is widely performed [16] In addition, a pattern of care study has demonstrated its steadily increasing use in North America [17]. However, the conventional brachytherapy planning has only used simple orthogonal $\mathrm{X}$-rays to evaluate the position of the applicator in relation to musculoskeletal pelvic anatomy and to prescribe the dose to a fixed reference point $\mathrm{A}$ ( $2 \mathrm{~cm}$ superior and $2 \mathrm{~cm}$ lateral to the distal end of the applicator/tandem), independently of specific tumor geometry or the individual patient anatomy [18]. Computed tomography (CT)-based conformal external beam radiotherapy planning defines nowadays the standard and is widely used in the majority of radiotherapy facilities throughout the world. In contrast, brachytherapy treat- ment planning based on 3D imaging volumetry is still not standard.

However, CT-based planning in brachytherapy has important advantages: 1) gapless volume planning; 2) precise applicator geometry reconstruction; 3) density information with the possibility to avoid high density gradients; 4 ) accurate measurement of all relative anatomical details in relation to the individual applicator geometry and spatial orientation.

Therefore, this first major UCP project was aimed to introduce and validate at Chiang Mai University CT-based adaptive intracavitary brachytherapy for locally advanced cervical cancer as a first step of image-guidance development. This new approach was based on the recently published European guidelines for three-dimensional ICBT for cervical cancer by the Groupe Européen de Curiethérapie - European Society for Therapeutic Radiology and Oncology (GEC-ESTRO) $[19,20]$. With respect to the discussed local limited resources, the study focused on patients who would potentially most benefit from this novel therapy protocol and the inclusion criteria were defined accordingly: histologically confirmed cervical carcinoma, younger age (20 to 60 years at therapy entry), good Karnofsky performance status, locally advanced tumors (FIGO stages IB2 to IIIB), no emergency conditions, no history of allergy (contrast media or chemotherapy), and informed consent. Exclusion criteria were: age at therapy entry $>60$ years, metastatic disease, poor underlying conditions, allergy to used chemotherapy agents or contrast media, or poor Karnofsky performance status (KI $\leq 70 \%)$. The study was initiated after obtaining an ethical votum from the local university commission and following the installation of a radiotherapy-dedicated CT-scanner in the department. The study treatment protocol consisted of CT-based 3D conformal external beam radiotherapy (EBRT) of the pelvis to $50 \mathrm{~Gy}$ in 25 fractions and 4 fractions of image-guided adaptive (CT-based) IC-BT of 6.5 to $7 \mathrm{~Gy}$. Concurrent chemotherapy with weekly cisplatin $40 \mathrm{mg} / \mathrm{m}^{2}$ was also performed. From July 2008 and April 2010, twenty-two consecutive patients with histologically proven cervical cancer were referred to department of radiotherapy at Chiang Mai University for definitive treatment consisting of concurrent chemo-radiotherapy, according to the department protocol during the study time. Five patients had to be excluded from the trial due to emergency conditions (severe bleeding during EBRT). Seventeen patients were enrolled into this phase II trial. Median age was 50 years (range: $36-60)$, Karnofsky performance status was $80-100 \%$, and the majority of patients had large tumors (FIGO IIB $n=12$, and IIIB $n=4$, five patients had a tumor larger than $5 \mathrm{~cm}$ ). Brachytherapy planning started with a conventional point A-based calculation, which was then optimized using CT data sets with inserted brachytherapy applicator, and was performed by the PLATO planning system (Nucletron B.V., Veenendaal, The Netherlands). The cumulative prescribed dose of EBRT 50 Gy and 4 x 6.5-7 Gy IC-BT corresponded to the $\mathrm{BED}_{2 \mathrm{~Gy}}$ (equivalent dose in 2-Gy fractions) of $80 \mathrm{~Gy}$, and was calculated using the linear-quadratic model and assuming $\alpha / \beta$ ratio $=10$ for tumor and $\alpha / \beta=3$ for OARs (organs at risk). 
The used dose constraints were $\mathrm{D}_{90}$ minimum $90 \%$ for the high-risk clinical target volume (HR-CTV) according the GEC-ESTRO definition $[19,20]$, and for the OARs total $D_{2 c c}$ were 75 Gy $\alpha / \beta-3$ for rectum and sigmoid colon, and 90 Gy $\alpha / \beta-3$ for the bladder, respectively. The definition for $D_{2 c c}$ being the minimum doses calculated at the most irradiated $2 \mathrm{cc}$ volumes.

The published results demonstrated the clear superiority of adaptive CT-based planning versus conventional methods. While the target coverage in terms of $\mathrm{D}_{90} \mathrm{HR}-$ CTV was excellent (but not different than conventional planning, $p=0.16$ ), the OARs (sigmoid and bladder) radiation exposure was significantly reduced $(p<0.001)$, when CT-based planning was used providing very low late toxicity with only 2 cases of gastrointestinal grade $\geq 3$ morbidity [21]. In addition, after a follow-up of 19 months no tumor relapse was observed.

\section{Second University Cooperation Platform major project - introduction of MRI-based image guided cervix brachytherapy}

Complementary to the advantages of CT-based volume planning in brachytherapy, magnetic resonance imaging (MRI) adds the better soft tissue information and enables in consequence a more accurate target definition. However, the logistics are more complicated and may differ considerably from using CT alone for brachytherapy guidance and planning. Encouraged by the publication of Viswanathan et al. [22], the introduction of MRI planning in cervical brachytherapy at the Radiotherapy Department in Chiang Mai was the main goal of this second UCP major project. However, a dedicated MRI scanner for radiotherapy did not exist. Thus, the use of MRI imaging had to be adapted to the local scarce availability and restricted to the time points of maximal potential benefit for the patient along with the minimum of disturbance in department logistics and interdisciplinary use of institutional resources. In consequence, three important time point were defined: 1) at diagnosis and prior to treatment in order to determine more accurately the IRand HR-CTV target volumes according to GEC-ESTRO definitions $[19,20]$; 2) after completion of external beam radiotherapy for the planning of the first brachytherapy fraction supplementary to $\mathrm{CT}$, and 3) three months after treatment for the first follow-up restaging. The feasibility and valence of restricted MRI single use for the first brachytherapy fraction is also confirmed by the Aarhus University experience demonstrating significant tumor volume shrinkage during external beam radiotherapy, but a rather constant target volume after EBRT completion and during the course of brachytherapy [23].

From February 2009 to March 2011, fifteen patients with cervical cancer were included in this study incorporating MRI at diagnosis, at first brachytherapy for planning in addition to CT, and three months after therapy. Inclusion and exclusion criteria were identical as in the CT-based planning study focusing on large cervical tumors (FIGO IIB $n=13$, IIIB $n=2$, and nine patients had a tumor larger than $5 \mathrm{~cm}$ ). In addition, all proven brachytherapy constraints for HR-CTV and OARs (see first UCP major project) were adopted. The results of this study confirmed the significant reduction in critical organs, which was even more pronounced than in the prior CT trial in bladder $(p=0.003)$, rectum $(p=0.015)$, and sigmoid $(p=0.012)$, while HR-CTV doses could be maintained > 95 Gy EQD2 [24]. The acute and late toxicity was excellent, as well as without observed grade 3 or higher morbidity. The addition of MRI imaging into the brachytherapy planning process was very feasible in terms of clinical tolerance, but added significantly more time with 3 to 4 hours of total treatment fraction time in comparison with conventional planning 0.5-1 hour, and 1-2 hours in CT based brachytherapy.

\section{Third University Cooperation Platform major project - combination of image guided adaptive brachytherapy and high technology external beam radiotherapy}

The next logical step of development at Chiang Mai University was the implementation of high-technology combination of image-guided brachytherapy (IGBT) with intensity modulated external beam radiotherapy (IMRT) in the treatment of locally advanced cervical cancer, since a series of prior publications demonstrated the superiority of IMRT versus 3D conformal external beam radiotherapy in reducing the OARs doses [25, 26].

Fifteen patients with locally advanced cervical cancer were enrolled into this additional phase II study combining whole-pelvis intensity modulated external beam radiotherapy (WP-IMRT) to a total dose of 45 Gy in 25 fractions and image-guided brachytherapy (IGBT) using CT was performed at the dose of $7 \mathrm{~Gy} \times 4$ fractions to the highrisk clinical target volume (HR-CTV). Again, the CT-trial inclusion and exclusion criteria were reused maintaining the study focus on large cervical tumors (FIGO IIB $n=12$, IIIB $n=3$ ), and the established brachytherapy constraints for HR-CTV and OARs (see first and second UCP major projects) were re-adopted. This study reconfirmed the prior findings of significant OARs dose reduction in bladder $(p<0.001)$, rectum $(p=0.001)$, and sigmoid $(p<0.001)$, and maintained a high curative HR-CTV total dose (mean EQD2: 88 Gy) [27].

In addition, thirty-nine patients were evaluated by rectosigmoidoscopy for late rectal toxicity in a retrospective study in patients treated with IGBT for cervical cancer demonstrating a clear association between measured cumulative rectal dose in EQD2 > 65 Gy and grade $\geq 2$ LENT-SOMA late morbidity [28]. These findings would encourage further efforts to minimize the OARs doses.

\section{Fourth University Cooperation Platform major project - addition of ultrasound in image-guided brachytherapy guidance and planning}

Despite the added benefits of magnetic resonance imaging to CT-based volume planning in cervical brachytherapy, MRI is still representing a limited resource, complicates significantly the department logistics, and is 
not offering an imaging guidance in real time. In contrast, ultrasound has these advantages of intraoperative, real time image-guidance and availability, which have defined this option as method of choice in adaptive prostate brachytherapy [29]. The use of ultrasound in gynecological brachytherapy also provides the advantage of soft tissue information in addition to the most used orthogonal X-ray imaging to assess intracavitary implant geometry in relation to the surrounding anatomy along with portability and cost-effectiveness [30].

The first three UCP projects documented a significant improvement in image-guided cervical cancer brachytherapy, but were associated with strict patient volume limitations due to the discussed resource situation. Thus, the implementation of transabdominal ultrasound (TAUS) was the focus of the fourth UCP project aiming a more generous potential use of image-guidance on long-term, and enhancing the quality of soft tissue assessment in gynecological brachytherapy. From February 2012 to December 2012, twenty-nine patients with FIGO IB-IVA cervical cancer (FIGO IIB $n=17$, and IIIB $n=9$ ) were consecutively treated and analyzed by radical combined radiotherapy consisting of whole-pelvis external beam radiotherapy (WP-EBRT) to 50 Gy (25 x 2 Gy), with central shielding after completion of 44 Gy and TAUS-guided intracavitary brachytherapy to the total cervical dose of EQD2 $\geq 80 \mathrm{~Gy}$, while maintaining low doses to the ICRU report no. 38 bladder and rectal points. The study focused on survival outcomes and toxicity profiles. The trial revealed TAUS-guided plans reduced significantly the OARs dose in bladder and rectum with a total EQD2 > 80 Gy for bladder in only $17.2 \%$ versus $62.1 \%$ in conventional planning, and EQD2 > 75 Gy for rectum in $44.8 \%$ versus $79.3 \%$. After a median follow-up (FU) time of 19 months, the local control, disease-free survival and overall survival estimates according to Kaplan-Meier were 93.1\%, 86.2\%, and 96.6\%, respectively. In addition, no grade 3 and higher toxicity was observed during the entire follow-up period [31].

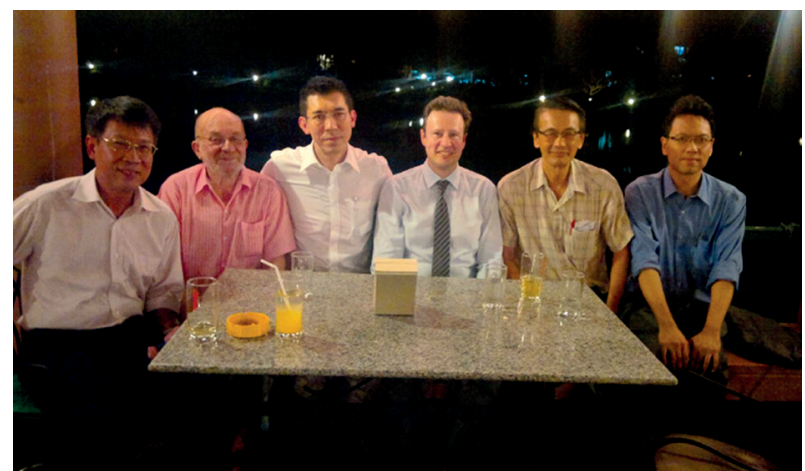

Fig. 1. Members of University Cooperation Platform (UCP) between the Christian-Albrechts-University Kiel (Germany) and Chiang Mai University (Thailand). From left to right: Somsak Wanwilairat (physicist), Dieter Hebbinghaus (computer scientist), Apichart Panichevaluk (radiation oncologist), Razvan Galalae (radiation oncologist), Vicharn Lorvidhaya (radiation oncologist), and Ekkasit Tharavichitkul (radiation oncologist)

\section{Review of the University Cooperation Platform experience}

Judging the entire cooperation experience it can be stated that the intermediate-term analyses of high-doserate IGBT in patients with advanced gynecological cancer revealed excellent results in terms of tumor control, survival, and treatment related long-term toxicity. Magnetic resonance imaging (MRI) based adaptive BT is the golden standard in terms of HR-CTV definition and soft tissue assessment. However, the combination of high-technology external beam radiotherapy (IMRT/Tomo) with CT-based IGBT is easier to manage in a very high volume institution as Chiang Mai University. In general, however, CT/MRI based image-guidance in gynecological brachytherapy alters the department logistics significantly. Patient selection and productivity remain an addressed, but not completely solved problem. To create a well balanced situation between time/cost effectivity and treatment quality is the crucial goal of technical development on this field. In this context, TAUS-based guidance adds quality aspects to the "classical" conventional X-ray based planning especially in terms of realtime measures and adequate soft tissue information, and may improve significantly the dose burden in OARs. TAUS-based BT actually refines conventional brachytherapy, and could be considered an intermediate step between conventional and CT/MRI based brachytherapy. In conclusion, making the different image guidance options (from US, to CT and MRI) disposable and created also the possibility to cherry pick more tailored treatment solutions in individual patients in the context of the actual department work load and available resources.

And finally, the UCP experience was a success story demonstrating that know-how and technology transfer was possible, and turned out to be not unidirectional, but rather bidirectional, as actually intended, to the very best interest of both involved institutions. In addition, knowledge and scientific evidence accumulation was also possible within these consecutive phases of technology and novel clinical protocol implementations, despite learning curves and logistical obstacles, and converted a high patient volume department into a regional/international teaching institution.

\section{Future University Cooperation Platform plans}

This established University Cooperation Platform strives for the next stages of development as part of a "visiting professor" program in August 2015 in Chiang Mai. E.g. the implementation of image-guided adaptive brachytherapy for prostate cancer and preparatory work for breast brachytherapy embedded in sustainable intensity modulated external beam protocols are the next planned projects.

\section{Razvan Galalae - personal acknowledgements}

First and foremost, I would like to mention and very much appreciate the preliminary and very important work of the Kiel University physicist Professor Henning 
Wendhausen, who unfortunately passed away. He actually initiated the University of Kiel cooperation with different Thai institutions within an IAEA (International Atomic Energy Agency) frame.

In general, I would like to express my sincere gratitude to the entire teams of the radiotherapy departments in Chiang Mai and Kiel Universities. In this context, the Kiel physicists Peter Kohr and Martin Jensen, the computer scientist Dieter Hebbinghaus, and the Chiang Mai physicist Somsak Wanwilairat have played outstanding roles making the UCP plans all possible. The radiation oncologists Pimkwan Kamnerdsupaphon, Ekkasit Tharavichitkul, and Apichart Panichevaluk from Chiang Mai University have became not only close cooperation partners, but also close scientific fellows. Special thanks are to be dedicated to the Chiang Mai University radiation oncologist Imjai Chitapanarux, and the Kiel radiation oncologists Jürgen Schultze for their continuous support, as well. Figure 1 depicts some members of the University Cooperation Platform Chiang Mai - Kiel. It is worth to mention the development of many close friendships among members of this cooperative association.

Finally, I would like to thank sincerely Professor Bernhard Kimmig and the computer scientist Dieter Hebbinghaus for believing in my vision of intercultural research and clinical cooperation and helping me continuously to implement it.

Professor Vicharn Lorvidhaya was, is, and will remain the most distinguished, prestigious, honorable, and reliable colluege I ever met. I consider myself very privileged being in the position to conduct together with Professor Lorvidhaya this rewarding work more than one decade.

\section{Disclosure}

Authors report no conflict of interest.

\section{References}

1. Ferlay J, Bray F, Pisani P, Parkin DM. GLOBOCAN 2002: Cancer incidence, mortality and prevalence worldwide. IARC Cancer Base No. 5, version 2.0. IARC Press, Lyon 2004.

2. Vatanasapt V, Sriamporn S, Vatanasapt P. Cancer control in Thailand. J Clin Oncol 2002; 32: 82-91.

3. Rose PG, Bundy BN, Watkins EB et al. Concurrent cisplatinbased radiotherapy and chemotherapy for locally advanced cervical cancer. N Engl J Med 1999; 340: 1144-1153.

4. Keys HM, Bundy BN, Stehman FB et al. Cisplatin, radiation, and adjuvant hysterectomy compared with radiation and adjuvant hysterectomy for bulky stage IB cervical carcinoma. N Engl J Med 1999; 340: 1154-1161.

5. Eifel PJ, Winter K, Morris M et al. Pelvic irradiation with concurrent chemotherapy versus pelvic and para-aortic irradiation for high-risk cervical cancer: An update of Radiation Therapy Oncology Group trial (RTOG) 90-01. J Clin Oncol 2004; 22: 872-880

6. Whitney CW, Sause W, Bundy BN et al. A randomized comparison of fluorouracil plus cisplatin versus hydroxyurea as an adjunct to radiation therapy in stages IIB-IVA carcinoma of the cervix with negative para-aortic lymph nodes: A Gynecologic Oncology Group and Southwest Oncology Group study. J Clin Oncol 1999; 17: 1339-1348.

7. Peters WA III, Liu PY, Barrett RJ II et al. Concurrent chemotherapy and pelvic radiation therapy compared with pelvic radiation therapy alone as adjuvant therapy after radical surgery in high-risk early-stage cancer of the cervix. J Clin Oncol 2000; 18: 1606-1613.

8. Fu KK. Biological basis for the interaction of chemotherapeutic agents and radiation therapy. Cancer 1985; 55 (9 Suppl): 2123-2130.

9. Pötter R, Dimopoulos J, Georg P et al. Clinical impact of MRI assisted dose volume adaptation and dose escalation in brachytherapy of locally advanced cervix cancer. Radiother Oncol 2007; 83: 148-155.

10. IARC. Cervix cancer screening: IARC handbook of cancer prevention. Vol. 10. IARC, Lyon 2005.

11. Galalae R. Kiel maintains close contacts with Chiang Mai. Profil UKSH 2003; 3: 5; available at: http:/ / biz.uk-sh.de/media/ custom/676_225_3.PDF

12. Chitapanarux I, Lorvidhaya V, Kamnerdsupaphon P et al. Chemoradiation comparing cisplatin versus carboplatin in locally advanced nasopharyngeal cancer: randomised, non-inferiority, open trial. Eur J Cancer 2007; 43: 1399-1406.

13. Tharavichitkul E, Pinitpatcharalerd A, Lorvidhaya V et al. Impact of incomplete plan to treatment results of concurrent weekly cisplatin and radiotherapy in locally advanced cervical cancer. J Radiat Res 2011; 52: 9-14.

14. Tharavichitkul E, Klunkin $\mathrm{P}$, Lorvidhaya $\mathrm{V}$ et al. The effects of two HDR brachytherapy schedules in locally advanced cervical cancer treated with concurrent chemoradiation: a study from Chiang Mai, Thailand. J Radiat Res 2012; 53: 281-287.

15. Stewart AJ, Viswanathan AN. Current controversies in highdose-rate versus low-dose-rate brachytherapy for cervical cancer. Cancer 2006; 107: 908-915.

16. Nag S, Orton C, Young D et al. The American Brachytherapy Society Survey of brachytherapy practice for carcinoma of the cervix in the United States. Gyn Oncol 1999; 73: 111-118.

17. Eifel PJ, Moughan J, Erickson B et al. Patterns of radiotherapy practice for patients with carcinoma of the uterine cervix: a patterns of care study. Int J Radiat Oncol Biol Phys 2004; 60: 1144-1153.

18. International Commission on Radiation Units and Measurements (ICRU). Dose and volume specifications for reporting intracavitary therapy in gynecology. ICRU Report no. 38. ICRU, Bethesda 1985.

19. Haie-Meder C, Pötter R, Van Limbergen E et al. Recommendations from Gynaecological (GYN) GEC-ESTRO Working Group (I): Concepts and terms in 3D image based 3D treatment planning in cervix cancer brachytherapy with emphasis on MRI assessment of GTV and CTV. Radiother Oncol 2005; 74: 235-245.

20. Pötter R, Haie-Meder C, Van Limbergen E et al. Recommendations from Gynaecological (GYN) GEC ESTRO working group (II): Concepts and terms in 3D image-based treatment planning in cervix cancer brachytherapy-3D dose volume parameters and aspects of 3D. Radiother Oncol 2006; 78: 67-77.

21. Tharavichitkul E, Mayurasakorn S, Lorvidhaya V et al. Preliminary results of conformal computed tomography (CT)based intracavitary brachytherapy (ICBT) for locally advanced cervical cancer: a single institution's experience. J Radiat Res 2011; 52: 634-640.

22. Viswanathan AN, Dimopoulos J, Kirisits C et al. Computed tomography versus magnetic resonance imaging-based contouring in cervical cancer brachytherapy: results of a prospective trial and preliminary guidelines for standardized contours. Int J Radiat Oncol Biol Phys 2007; 68: 491-498.

23. Fokdal L, Tanderup K, Hockland SB et al. Clinical feasibility of combined intracavitary/interstitial brachytherapy in locally advanced cervical cancer employing MRI with a tan$\mathrm{dem} /$ ring applicator in situ and virtual preplanning of the interstitial component. Radiother Oncol 2013; 107: 63-68. 
24. Tharavichitkul E, Sivasomboon C, Wanwilairat S et al. Preliminary results of MRI-guided brachytherapy in cervical carcinoma: the Chiang Mai University experience. J Radiat Res 2012; 53: 313-318.

25. Mundt AJ, Lujan AE, Rotmensch J et al. Intensity-modulated whole pelvic radiotherapy in women with gynecologic malignancies. Int J Radiat Oncol Biol Phys 2002; 52: 1330-1337.

26. Hasselle MD, Rose BS, Kochanski JD et al. Clinical outcomes of intensity-modulated pelvic radiation therapy for carcinoma of the cervix. Int J Radiat Oncol Biol Phys 2011; 80: 14361445.

27. Tharavichitkul E, Wanwilairat $S$, Chakrabandhu $S$ et al. Image-guided brachytherapy (IGBT) combined with whole pelvic intensity-modulated radiotherapy (WP-IMRT) for locally advanced cervical cancer: a prospective study from Chiang Mai University Hospital, Thailand. J Contemp Brachytherapy 2013; 5: 10-16.

28. Tharavichitkul E, Meungwong $\mathrm{P}$, Chitapanarux $\mathrm{T}$ et al. The association of rectal equivalent dose in 2 Gy fractions (EQD2) to late rectal toxicity in locally advanced cervical cancer patients who were evaluated by rectosigmoidoscopy in Faculty of Medicine, Chiang Mai. Radiat Oncol J 2014; 32: 57-62.

29. Grills IS, Martinez AA, Hollander M et al. High dose rate brachytherapy as prostate cancer monotherapy reduces toxicity compared to low dose rate palladium seeds. J Urol 2004; 171: 1098-1104.

30. van Dyk S, Schneider M, Kondalsamy-Chennakesavan S et al. Ultrasound use in gynecologic brachytherapy: time to focus the beam. Brachytherapy 2015, Jan 22; DOI: 10.1016/j.brachy. 2014.12.001 [Epub ahead of print].

31. Tharavichitkul E, Tippanya D, Jayavasti R et al. Two-year results of transabdominal ultrasound-guided brachytherapy for cervical cancer. Brachytherapy 2014 Dec 4; DOI: 10.1016/j. brachy.2014.11.001 [Epub ahead of print] 